MATHEMATICS OF COMPUTATION

Volume 70, Number 236, Pages 1699-1705

S $0025-5718(00) 01291-6$

Article electronically published on October 18, 2000

\title{
DENSITY OF CUBIC FIELD DISCRIMINANTS
}

\author{
DAVID P. ROBERTS
}

\begin{abstract}
In this paper we give a conjectural refinement of the DavenportHeilbronn theorem on the density of cubic field discriminants. Our refinement is plausible theoretically and agrees very well with computational data.
\end{abstract}

\section{INTRODUCTION}

Let $a_{n}$ be the number of isomorphism classes of abelian cubic fields with discriminant $n$. Let $b_{n}$ be the number of isomorphism classes of nonabelian cubic fields with discriminant $n$. The numbers $a_{n}$ are very well understood. The numbers $b_{n}$ have been the subject of extensive theoretical and computational study for at least sixty years, but are less well understood. The object of this note is to contribute to the study of these $b_{n}$ by bringing together the theoretical and computational literature.

For $\alpha \in\{-,+\}$, define

$$
g_{\alpha}(x)=\sum_{n=1}^{x} b_{\alpha n} .
$$

Put $C_{-}=3$ and $C_{+}=1$. The main theoretical result is due to Davenport and Heilbronn [8]:

$$
g_{\alpha}(x) \sim C_{\alpha} \frac{1}{12 \zeta(3)} x .
$$

The numbers $b_{\alpha n}$ have been computed for larger and larger $n$ by many authors. The most recent results are due to Belabas [3], who introduced a vastly improved method and worked through the cutoff $x=10^{11}$.

It has been discussed by several authors that the "match" between computational evaluation of $g_{\alpha}(x)$ and the asymptotic formula (1.2) is not very good, as illustrated by Tables 1, 2, and 3 below. For example, the paper [9] dealt with the case $\alpha=-$; here is an excerpt from pages $322-324$, with some trivial notational changes:

If, however, the reader were to plot the density versus $x$, he would be somewhat astonished to see that this density is increasing so slowly that his first impression would be that it will not make it to the DavenportHeilbronn limit. Thus it remains a challenging problem, assuming that the $\mathrm{D}-\mathrm{H}$ limit is not in error, to explain the origin of this very slow convergence. This problem was indicated by Shanks in [14, 13, and on the real side in [14, [10, where the problem is further aggravated

Received by the editor April 20, 1999 and, in revised form, January 6, 2000.

2000 Mathematics Subject Classification. Primary 11N56, 11R16.

(C)2000 American Mathematical Society 
by even slower convergence. To date, and to our knowledge, no good quantitative explanation of this phenomenon has been given.

In this paper we conjecture a quantitative explanation of this slow-convergence phenomenon.

\section{The ABelian CASE}

It is best to briefly review the situation in the abelian case. One has the formula

$$
\sum_{n=1}^{\infty} \frac{a_{n}}{n^{s}}=-1+\left(1+\frac{2}{81^{s}}\right) \prod_{p \equiv 1(6)}\left(1+\frac{2}{p^{2 s}}\right) .
$$

Put

$$
f(x)=\sum_{n=1}^{x} a_{n}
$$

Cohn [4] showed that the function (2.1) continues analytically to $\operatorname{Re}(s)>1 / 4$, except for a simple pole at $s=1 / 2$. He computed the residue of this pole. This gave him more than enough information to conclude that

$$
f(x) \sim\left(\frac{11 \sqrt{3}}{36 \pi} \prod_{p \equiv 1(6)} \frac{(p+2)(p-1)}{p(p+1)}\right) x^{1 / 2} .
$$

Define $h_{-}(x)=g_{-}(x)$ and

$$
h_{+}(x)=g_{+}(x)+\frac{1}{3} f(x) .
$$

From (1.2) and (2.3) one sees that (1.2) would remain true with $g_{\alpha}$ replaced by $h_{\alpha}$. We henceforth focus more on $h_{\alpha}$, as it appears naturally in (3.2) and (3.3).

\section{The Basic COnjeCture}

Belabas proved [3, (8) and (20)] that, for either $\alpha$, the difference between the sides of (1.2) is $o\left(x /(\log x)^{2}\right)$. From his extensive computational data he speculated [3, page 1232] that these differences are $o\left(x /(\log x)^{\beta}\right)$ for arbitrarily large $\beta$. We believe a stronger statement. Recall that $C_{-}=3$ and $C_{+}=1$. Put $K_{-}=\sqrt{3}$ and $K_{+}=1$.

Conjecture 3.1. For each $\alpha$,

$$
h_{\alpha}(x)=C_{\alpha} \frac{1}{12 \zeta(3)} x+K_{\alpha} \frac{4 \zeta(1 / 3)}{5 \Gamma(2 / 3)^{3} \zeta(5 / 3)} x^{5 / 6}+o\left(x^{5 / 6}\right) .
$$

Numerically, 3.1) works out to

$$
\begin{aligned}
& h_{-}(x)=(0.2079768431 \ldots) x-(0.2557983756 \ldots) x^{5 / 6}+o\left(x^{5 / 6}\right), \\
& h_{+}(x)=(0.0693256143 \ldots) x-(0.1476852610 \ldots) x^{5 / 6}+o\left(x^{5 / 6}\right) .
\end{aligned}
$$

Note that the coefficient of $x^{5 / 6}$ is negative in each case, the negative sign coming from $\zeta(1 / 3)<0$. This negativity corresponds to the fact that computationally one has always found fewer fields than the Davenport-Heilbronn asymptotic would suggest.

The heuristic we are about to sketch is too crude to suggest any particular error term in (3.1). The $o\left(x^{5 / 6}\right)$ we have written there corresponds to the weakest 
conjecture in which our conjectured new term actually plays a role. However, Tables 1 and 2 below suggest that the error term should be reducible at least most of the way to $o\left(x^{1 / 2}\right)$.

We are led to our conjecture theoretically as follows. Define a Dirichlet series

$$
\xi_{\alpha}(s)=\sum_{k \in \mathcal{K}_{\alpha}} \frac{1}{|\operatorname{Aut}(k)|\left|D_{k}\right|^{s}} .
$$

Here $\mathcal{K}_{\alpha}$ is the set of isomorphism classes of cubic fields $k$ with discriminant of sign $\alpha$. Also, $D_{k}$ is the discriminant of $k$ while $\operatorname{Aut}(k)$ is the automorphism group of $k$; so $|\operatorname{Aut}(k)|$ is 3 or 1 according to whether $k$ is abelian or not, the former case occuring only for $\alpha=+$.

For $S$ a finite set of primes, define a weighted version of (3.2):

$$
\xi_{\alpha, S}(s)=\sum_{k \in \mathcal{K}_{\alpha}} \frac{\eta_{k, S}(s)}{|\operatorname{Aut}(k)|\left|D_{K}\right|^{s}} .
$$

Here the weights $\eta_{k, S}(s)$ are given in [6] page 122]. These weights are themselves Dirichlet series $\sum c_{m}(k, S) m^{-s}$ with integral coefficients $c_{m}(k, S)$ decreasing to the Kronecker symbol $\delta_{m 1}$ as $S$ grows. Thus $\xi_{\alpha, S} \rightarrow \xi_{\alpha}$ in the sense of formal Dirichlet series.

The point of introducing the approximations $\xi_{\alpha, S}$ is that one understands them analytically better than one understands $\xi_{\alpha}$ itself. From Davenport's earlier work [7] one knows that the right-most pole of $\xi_{\alpha, \emptyset}$ is at $s=1$. The analytic behavior of $\xi_{\alpha, \emptyset}$ was investigated more explicitly by Shintani [15], who found the next pole at $s=5 / 6$. More generally, it is proved in [5, Prop. 6.2], that each $\xi_{\alpha, S}$ continues to the whole $s$-plane, meromorphically with only poles at $s=1$ and $s=5 / 6$. These poles are simple, their residues $C_{\alpha} r_{1, S}$ and $K_{\alpha} r_{5 / 6, S}$ being deducible from the first two equations in [5, Thm. 6.2i]. In the case $s=1$, one has to set $b_{\alpha}$ equal to zero in the corresponding equation; this $b_{\alpha}$ corresponds to quadratic fields, included in $\xi_{\alpha, S}$ in [5] and [6], but not included here.

From the formulas in [5. Thm. 6.2i], one sees that the numbers $r_{1, S}$ are positive, and decreasing as $S$ grows. Similarly, the numbers $r_{5 / 6, S}$ are negative and increasing as $S$ grows. Let $r_{1}$ and $r_{5 / 6}$ be their respective limits. One does not have enough control at present to rigorously apply any "poles-control-growth" theorems. However the general formalism suggests that $h_{\alpha}(x)$ grows as $C_{\alpha} r_{1} x+K_{\alpha}(6 / 5) r_{5 / 6} x^{5 / 6}$. This is the source of our Conjecture 3.1. The constant $r_{5 / 6}$ is actually nicer than we had initially expected from its source as the limit of the $r_{5 / 6, S}$; special zeta values arise because of the factorization of the right side of (5.3) below.

To follow our heuristic in a more detailed way, one should first understand it in the more refined context of Section 5 below. We recommend looking next at Prop. 2.1 and the discussion around it in [6]; this treats in more classical language the special case of [5, Thm 6.2i] we need, except it does not repeat the formula for $r_{5 / 6, S}$.

\section{NUMERICAL EVIDENCE FOR THE BASIC CONJECTURE}

Our conjecture for $\alpha=-$ compares with Belabas's data [3] page 1232] for negative-discriminant cubic fields as in Table 1 Here and below, $x=10^{j}$. Also $H_{\alpha}(x)$ denotes the right side of (1.2) while $H_{\alpha}^{*}(x)$ denotes the first two terms of the right side of (3.1). Clearly $H_{-}^{*}$ matches the data $h_{-}$substantially better than $H_{-}$ 
TABLE 1 . Imaginary cubic fields: Computation $h_{-}$and theory $H_{-}^{*}$

\begin{tabular}{|c|c|c|c|c|}
\hline$j$ & $h_{-}(x)$ & $\frac{h_{-}(x)}{H_{-}(x)}$ & $\frac{h_{-}(x)}{H_{-}^{*}(x)}$ & $\frac{h_{-}(x)-H_{-}^{*}(x)}{x^{1 / 2}}$ \\
\hline 2 & 7 & 0.337 & 0.7843510 & -0.1925 \\
\hline 3 & 127 & 0.611 & 0.9993210 & -0.0027 \\
\hline 4 & 1,520 & 0.731 & 0.9943300 & -0.0867 \\
\hline 5 & 17,041 & 0.819 & 0.9998781 & -0.0066 \\
\hline 6 & 182,417 & 0.877 & 1.0001096 & 0.0200 \\
\hline 7 & $1,905,514$ & 0.916 & 1.0000100 & 0.0060 \\
\hline 8 & $19,609,185$ & 0.943 & 0.9999394 & -0.1188 \\
\hline 9 & $199,884,780$ & 0.961 & 0.9999850 & -0.0951 \\
\hline 10 & $2,024,660,098$ & 0.974 & 1.0000009 & 0.0176 \\
\hline 11 & $20,422,230,540$ & 0.982 & 1.0000003 & 0.0218 \\
\hline
\end{tabular}

TABLE 2. Real cubic fields: Computation $h_{+}$and theory $H_{+}^{*}$

\begin{tabular}{r|rr|ccc|c}
$j$ & \multicolumn{1}{r}{$g_{+}(x)$} & $f(x)$ & $\frac{h_{+}(x)}{H_{+}(x)}$ & $\frac{h_{+}(x)}{H_{+}^{*}(x)}$ & $\frac{h_{+}(x)-H_{+}^{*}(x)}{x^{1 / 2}}$ & $\frac{g_{+}(x)-H_{+}^{*}(x)}{x^{1 / 2}}$ \\
\hline 2 & 0 & 2 & 0.096 & 8.5889786 & 0.0589 & -0.0078 \\
3 & 22 & 5 & 0.341 & 1.0461129 & 0.0330 & -0.0197 \\
4 & 366 & 16 & 0.536 & 0.9900166 & -0.0374 & -0.0908 \\
5 & 4,753 & 51 & 0.688 & 1.0010833 & 0.0163 & -0.0374 \\
6 & 54,441 & 159 & 0.786 & 0.9988436 & -0.0631 & -0.1161 \\
7 & 592,421 & 501 & 0.855 & 0.9999134 & -0.0162 & -0.0690 \\
8 & $6,246,698$ & 1,592 & 0.901 & 1.0000259 & 0.0161 & -0.0369 \\
9 & $64,654,353$ & 5,008 & 0.933 & 1.0000097 & 0.0198 & -0.0330 \\
10 & $661,432,230$ & 15,851 & 0.954 & 0.9999988 & -0.0081 & -0.0609 \\
11 & $6,715,773,873$ & 50,152 & 0.969 & 1.0000002 & 0.0046 & -0.0482
\end{tabular}

does. The poor match of $H_{-}$to $h_{-}$in the range $10^{5} \leq x \leq 10^{6}$ formed the basis of the quote in Section 1

For $\alpha=+$, we start from the values of $g_{+}(x)+f(x)$ listed in [3, page 1232]. We use (2.1) to compute $f(x)$ and thereby deduce $h_{+}(x)=g_{+}(x)+(1 / 3) f(x)$. Our hope that the error term in Conjecture 3.1 can be reduced to $o\left(x^{1 / 2}\right)$ would require that the numbers in the last column of Table 1 and the next to last column of Table 2 tend to zero. The data seems rather borderline in support of $o\left(x^{1 / 2}\right)$, but strongly indicative that our conjecture as stated is true, and moreover that the $o\left(x^{5 / 6}\right)$ can be reduced at least somewhat.

From Cohn's result (2.3), at most one linear combination $g_{+}(x)+u f(x)$ can agree with $H_{+}^{*}(x)$ to within $o\left(x^{1 / 2}\right)$. The last two columns of Table 2 suggest that $u=1 / 3$ is more likely than $u=0$. In fact $u=0.31 \ldots$ would give the best fit, in a least squares sense, and this agrees well with the theoretically favored $u=1 / 3$.

\section{The REFINED CONJECTURE}

The Davenport-Heilbronn theorem comes in a more refined version and so does our conjecture. Let $A$ be the set of symbols $\left\{111,21,3,1^{2} 1,1^{3}\right\}$. Then for $K$ a cubic field and $p$ a prime, the splitting behavior of $p$ in $K$ determines an element 
of $A$. If $p$ is unramified, then it either splits completely (111), splits partially (21), or remains inert (3). If $p$ is ramified, then it is either partially ramified $\left(1^{2} 1\right)$ or totally ramified $\left(1^{3}\right)$.

Until now, we have been using $\alpha$ to denote a sign, + or - . Henceforth, we use $\alpha$ to denote the following data. First, a finite set $\operatorname{support}(\alpha) \subset\{\infty, 2,3,5, \ldots\}$ of places of $\mathbf{Q}$, containing $\infty$. Second, a sign $\alpha_{\infty} \in\{+,-\}$, as before. Third, for each prime $p \in \operatorname{support}(\alpha)$, an element $\alpha_{p} \in A$. Let $\mathcal{K}_{\alpha}$ be the set of isomorphism classes of cubic fields meeting the given local condition $\alpha_{v}$ for each $v \in \operatorname{support}(\alpha)$. One naturally has $f_{\alpha}, g_{\alpha}, h_{\alpha}, \xi_{\alpha}$, and $\xi_{\alpha, S}$, all direct generalizations of the notions introduced above in the special case $\operatorname{support}(\alpha)=\{\infty\}$.

Define $C_{\infty,-}=3$ and $C_{\infty,+}=1$, as in Section 1 and $K_{\infty,-}=\sqrt{3}$ and $K_{\infty,+}=1$, as in Section 3. Define $p$-adic analogs as follows.

\begin{tabular}{l|ll}
$\alpha_{p}$ & $C_{p, \alpha_{p}}$ & \multicolumn{1}{c}{$K_{p, \alpha_{p}}$} \\
\hline 111 & $1 / 6 C_{p}$ & $\left(1+p^{-1 / 3}\right)^{3} / 6 K_{p}$ \\
21 & $1 / 2 C_{p}$ & $\left(1+p^{-1 / 3}\right)\left(1+p^{-2 / 3}\right) / 2 K_{p}$ \\
3 & $1 / 3 C_{p}$ & $\left(1+p^{-1}\right) / 3 K_{p}$ \\
$1^{2} 1$ & $1 / p C_{p}$ & $\left(1+p^{-1 / 3}\right)^{2} / p K_{p}$ \\
$1^{3}$ & $1 / p^{2} C_{p}$ & $\left(1+p^{-1 / 3}\right) / p^{2} K_{p}$
\end{tabular}

Here

$$
\begin{aligned}
C_{p} & =1+p^{-1}+p^{-2}, \\
K_{p} & =\frac{\left(1-p^{-5 / 3}\right)\left(1+p^{-1}\right)}{1-p^{-1 / 3}}
\end{aligned}
$$

are normalizing factors, chosen so that the column-sums $\sum C_{p, \alpha_{p}}$ and $\sum K_{p, \alpha_{p}}$ are both 1 . The values of $C_{p, \alpha_{p}}$ are taken from, e.g., [10, page 589] while the $K_{p, \alpha_{p}}$ are taken from [5, Table 5.1]. Note that both the $C_{p, \alpha_{p}}$ and the $K_{p, \alpha_{p}}$ are actually given by procedures uniform in $\alpha_{p}$. For example, the ratios $\left(K_{p, \alpha_{p}} K_{p}\right) /\left(C_{p, \alpha_{p}} C_{p}\right)$ are all given by replacing a factor $f^{e}$ in $\alpha_{p}$ by a factor $\left(1+p^{-f / 3}\right)$, e.g., $1^{2} 1 \mapsto$ $\left(1+p^{-1 / 3}\right)\left(1+p^{-1 / 3}\right)$.

We expect that Conjecture 3.1 holds verbatim for these more general $\alpha$, with

$$
\begin{aligned}
C_{\alpha} & =\prod_{v \in \operatorname{support}(\alpha)} C_{v, \alpha_{v}}, \\
K_{\alpha} & =\prod_{v \in \operatorname{support}(\alpha)} K_{v, \alpha_{v}} .
\end{aligned}
$$

Our heuristic continues to make sense in this more localized context. In fact the basic references [5] and [6] are in this context.

\section{Numerical EVIDEnCE for the Refined CONJECture}

In this section, we work with twenty different $\alpha$. All of them have support $\{\infty, p\}$ and $\alpha_{\infty}=+$. We let $p$ run over $\{2,3,5,7\}$ and let $\alpha_{p}$ run over the five-element set $A$. For simplicity, we compare computation with theory only at $x=10^{7}$.

We get the numbers $g_{\alpha}\left(10^{7}\right)$ by renormalizing the first four lines of [10, Table 11]. We get the numbers $f_{\alpha}\left(10^{7}\right)$ by a simple procedure based on (2.1). Including these extra 501 fields, with total mass $501 / 3=167$, improves the fit slightly, as would be expected from line $j=7$ of Table 2 
TABLE 3. Real cubic fields and their $p$-adic decomposition: Computation $h_{\alpha}$ and theory $H_{\alpha}^{*}\left(x\right.$ fixed at $\left.10^{7}\right)$

\begin{tabular}{r|cc|cc|cc|cc} 
& \multicolumn{2}{|c|}{$p=2$} & \multicolumn{2}{|c|}{$p=3$} & \multicolumn{2}{|c|}{$p=5$} & \multicolumn{2}{|c}{$p=7$} \\
$\alpha_{p}$ & $\frac{h_{\alpha}(x)}{H_{\alpha}(x)}$ & $\frac{h_{\alpha}(x)}{H_{\alpha}^{*}(x)}$ & $\frac{h_{\alpha}(x)}{H_{\alpha}(x)}$ & $\frac{h_{\alpha}(x)}{H_{\alpha}^{*}(x)}$ & $\frac{h_{\alpha}(x)}{H_{\alpha}(x)}$ & $\frac{h_{\alpha}(x)}{H_{\alpha}^{*}(x)}$ & $\frac{h_{\alpha}(x)}{H_{\alpha}(x)}$ & $\frac{h_{\alpha}(x)}{H_{\alpha}^{*}(x)}$ \\
\hline 111 & 0.706 & 0.9999 & 0.721 & 0.9998 & 0.734 & 0.9994 & 0.740 & 0.9994 \\
21 & 0.851 & 0.9999 & 0.856 & 1.0001 & 0.858 & 0.9996 & 0.858 & 0.9997 \\
3 & 0.924 & 1.0001 & 0.923 & 0.9999 & 0.920 & 1.0002 & 0.917 & 1.0008 \\
$1^{2} 1$ & 0.836 & 0.9996 & 0.835 & 0.9997 & 0.832 & 1.0002 & 0.829 & 0.9985 \\
$1^{3}$ & 0.909 & 1.0003 & 0.903 & 0.9998 & 0.895 & 1.0006 & 0.891 & 1.0025
\end{tabular}

Table 3 gives the comparison. This table illustrates how the slowness of the convergence $h_{\alpha}(x) / H_{\alpha}(x)$ to 1 seems to depend systematically on $\alpha_{p}$, the totally split case $\alpha_{p}=111$ being the slowest. On the other hand, $h_{\alpha}(x) / H_{\alpha}^{*}(x)$ is much closer to 1 , with no apparent tendency to be above or below.

\section{THREE CONCLUDING REMARKS}

By using the five element set $A$, we are falling slightly short of the most locally refined conjecture, because there are different ways a prime can be ramified. Thus, for example, if a field $K$ is $1^{3}$ at $p=3$, then there are actually nine possibilities for its 3 -adic completion $K_{3}$; both $C_{3,1^{3}}$ and $K_{3,1^{3}}$ should be split into nine parts, in the proportions indicated in the $\left(p, \alpha_{p}\right)=\left(3,1^{3}\right)$ slot in the following chart.

\begin{tabular}{c|ccc}
$\alpha_{p}$ & $p=2$ & $p=3$ & $p \geq 5$ \\
\hline $1^{2} 1$ & $\frac{1}{4} \frac{1}{4} \frac{1}{8} \frac{1}{8} \frac{1}{8} \frac{1}{8}$ & $\frac{1}{2} \frac{1}{2}$ & $\frac{1}{2} \frac{1}{2}$ \\
$1^{3}$ & 1 & $\frac{1}{3} \frac{1}{3} \frac{1}{9} \frac{1}{81} \frac{1}{81} \frac{1}{81} \frac{1}{81} \frac{1}{81} \frac{1}{81}$ & 1 or $\frac{1}{3} \frac{1}{3} \frac{1}{3}$
\end{tabular}

Similarly, if a field $K$ is $1^{3}$ at $p \geq 5$, there are either 1 or 3 possibilities for $K_{3}$, according to whether $p \equiv 5$, or 1 modulo 6 . In general, a $p$-adic algebra $K_{p}$ of type $\alpha_{p}$ contributes to $C_{p, \alpha_{p}}$ and $K_{p, \alpha_{p}}$ in proportion to $1 /\left(\left|\operatorname{Aut}\left(K_{p}\right)\right|\left|D_{K_{p}}\right|\right)$.

Our basic theoretical references [5] and [6] work over arbitrary number fields as well as finite extensions of $\mathbf{F}_{p}(t)$ for $p \geq 5$. We expect that the analog of our fully refined Conjecture 3.1 holds there too, but at present there is no corresponding published computational data.

The most important remaining problem is to prove our conjecture. The generally pessimistic discussion in [6, page 124] and [2, page 620] suggests to us that the way may be difficult. However, one ingredient of a proof might be the functional equation of $\xi_{\alpha, S}$ with respect to $s \mapsto 1-s$, studied in [15, [5], and [1]. Another ingredient might be [12, Thm. 3], which concerns growth of arithmetic functions whose associated Dirichlet series satisfy such a functional equation.

\section{REFERENCES}

[1] I. O. Angell, A table of totally real cubic fields, Math. Comput. 30 (1976), 184-187. MR 53:5528

[2] K. Belabas, Crible et 3-rang des corps quadratiques, Ann. Inst. Fourier (Grenoble), 46 (1996), 909-949. MR 98b:11112

[3] _ A fast algorithm to compute cubic fields, Math. Comp. 66 (1997), 1213-1237. MR 97m:11159 
[4] H. Cohn, The density of abelian cubic fields, Proc. Amer. Math. Soc. 5 (1954), 476-477. MR 16:222a

[5] B. Datskovsky and D. J. Wright, The adelic zeta function associated with the space of binary cubic forms, II: local theory, J. Reine Angew. Math. 367 (1986), 27-75. MR 87m:11034

[6] , Density of discriminants of cubic extensions, J. Reine Angew. Math. 386 (1988) 116-138. MR 90b:11112

[7] H. Davenport, On the class number of binary cubic forms, I \& II., J. London Math. Soc. 26 (1951), 183-198 (Corrigendum, ibid 27 (1952) 512). MR 13:323e, MR 13:323f

[8] H. Davenport and H. Heilbronn, On the density of discriminants of cubic fields (II), Proc. Roy. Soc. Lond. A 322 (1971) 405-420 MR 58:10816

[9] G. W. Fung and H. G. Williams, On the computation of complex cubic fields, with discriminant $D \geq-10^{6}$, Math. Comp. 55 (1990), 313-325. Errata Math. Comp. 63 (1994), 433. MR 94i: 11106

[10] P. Llorente and J. Quer, On totally real cubic fields with discriminant $d<10^{7}$, Math. Comp. 50 (1988), 581-594. MR 89g:11099

[11] Y. Ohno, A conjecture on coincidence among the zeta functions associated with the space of binary cubic forms, Amer. J. of Math. 119 (1997), 1083-1094. MR 98k:11037

[12] M. Sato and T. Shintani, On zeta functions associated with prehomogeneous vector spaces, Ann. of Math. 100 (1974), 131-170. MR 49:8969

[13] D. Shanks, Review of [1], Math. Comp. 30, (1976), 670-673.

[14] A survey of quadratic, cubic and quartic algebraic number fields (from a computational point of view), Cong. Numer. 17 (1976), 15-40. MR 56:11951

[15] T. Shintani, On Dirichlet series whose coefficients are class numbers of integral binary cubic forms, J. Math. Soc. Japan, 24 (1972), 132-188. MR 44:6619

Department of Mathematics, Rutgers University, Piscataway, New Jersey 08854

Current address: Division of Science and Mathematics, University of Minnesota-Morris, Morris, Minnesota 56267

E-mail address: roberts@mrs.umn.edu

$U R L:$ http://cda.mrs.umn.edu/ roberts/ 